PII S0038-1098(96)00266-9

\title{
DYNAMICAL SCREENING EFFECTS IN HOT-ELECTRON SCATTERING FROM ELECTRON-HOLE PLASMA AND LO-PHONON MODES IN QUANTUM WIRES
}

\author{
C.R. Bennett, ${ }^{a}$ B. Tanatar ${ }^{b}$ and N.C. Constantinou ${ }^{a}$ \\ ${ }^{a}$ Department of Physics, University of Essex, Colchester, CO4 3SQ, England \\ ${ }^{b}$ Department of Physies, Bilkent University, Bilkent, 06533 Ankara, Turkey
}

(Received 5 January 1996; accepted 25 May 1996 by R.T. Phillips)

\begin{abstract}
We present a fully dynamical and finite temperature study of the hotelectron momentum relaxation rate and the power loss in a coupled system of electron-hole plasma and bulk LO-phonons in a quantum wire structure. Interactions of the scattered electron with neutral plasma components and phonons are treated on an equal footing within the random-phase approximation. Coupled mode effects substantially change the transport properties of the system at low temperatures. Particularly, the "plasmon-like" and "LO-phonon-like" excitations yield comparable rates which, as a consequence of the singular nature of the 1D density of states, can be large at the threshold. This is in contrast to room temperature results where only the LOphonon mode contributes significantly to the rate. The density and temperature dependence of the power loss reveals that dynamical screening effects are important, and energy-momentum conservation cannot be satisfied above a certain density for a given initial energy. Copyright (C) 1996 Elsevier Science Ltd
\end{abstract}

\section{INTRODUCTION}

Electronic devices making use of the hot-electron scattering phenomenon have been a subject of interest for some time [1]. In particular, hot-electron transistors with a base region made of high-mobility semiconducting material like GaAs offer a high-speed device. Theoretical work on the inelastic scattering rates of such systems for zero and finite temperature in various approximation schemes has been presented $[2,3]$. Relatively few studies [4] are devoted to the scattering and energy-loss rates of hot-electrons from neutral plasmas as occur in photoexcited electronhole systems. The purpose of this paper is to investigate the momentum relaxation rate and power loss of hot-electrons in a photoexcited quasi-one-dimensional (Q1D) structure. It has been noted and discussed in detail by $\mathrm{Hu}$ and Das Sarma [5] that the momentum relaxation rate is a more meaningful quantity to study the hot-electron scattering in quantum wires. Plasmon phonon coupling in Q1D structures were recently studied by Hwang and Das Sarma
[6] and Bennett et al. [7]. We shall consider a quantum wire under intense photoexcitation, so that an equal number of electrons and holes are created, and scattering of electrons from the neutral plasma and from the LO-phonons will be taken into account. We have recently evaluated the momentum relaxation rate for the coupled plasmon-LO-phonon system; our conclusions are that coupled mode effects drastically change the transport properties of Q1D systems, especially at low temperatures [7].

\section{THEORY}

There are several different formulations [8] of the hot-electron scattering phenomenon. We assume a test electron to be injected into the quantum wire without modifying the properties of the coupled electron-hole-phonon system. We adopt the viewpoint of Das Sarma et al. [8] and use the standard electronscattering theory [9] treating the system to be not completely isolated. The coupled system is in quasiequilibrium and interacts with an external heat bath 
allowing phonons to decay with some phenomenological parameter. The main assumptions that go into our calculations are as follows.

(i) The electrons and holes are in their respective lowest subbands. We only consider the heavy holes, for simplicity.

(ii) The electrons and holes are in a quasi-equilibrium state interacting with each other via the bare Coulomb interaction, $V_{q}$. Neutral plasma components interact with LO-phonons via the Fröhlich interaction. We neglect phonon confinement effects.

(iii) Screening effects among the plasma components are treated within the random-phase approximation (RPA) where we also include the finite-temperature effects. We describe the hotelectron scattering within the Born approximation where the differential scattering rate is given by

$P(q, \omega)=-2 V_{q}^{\infty} \operatorname{Im}\left[\frac{1}{\epsilon_{T}(q, \omega)}\right] \frac{1}{1-\mathrm{e}^{-\beta \omega}}$,

in terms of the imaginary part of the total dielectric function of the neutral plasma-phonon system, $\epsilon_{T}(q, \omega)$. Here $\beta=\left(k_{B} T\right)^{-1}$ is the inverse of the lattice temperature. The above expression gives the probability of an electron with momentum $k$, transferring a momentum $q$ and energy $\omega$ to the coupled plasma-phonon system. The total scattering rate of a hot-electron with momentum $k$ is

$$
\begin{aligned}
\Gamma_{k}= & \int \frac{\mathrm{d} q}{2 \pi} \frac{q}{k} \int \mathrm{d} \omega P(q, \omega) \delta\left(\omega+k q / m-q^{2} / 2 m\right) \\
& \times[1-f(k-q)],
\end{aligned}
$$

where the $\delta$-function guarantees energy-momentum conservation in the scattering event, and the statistical factor describes the Pauli principle $[f(k)$ is the equilibrium Fermi distribution function]. The total dielectric function for the coupled neutral plasma-phonon system is written as

$\epsilon_{T}(q, \omega)=1+\frac{\omega_{\mathrm{LO}}^{2}-\omega_{\mathrm{TO}}^{2}}{\omega_{\text {TO }}^{2}-\omega^{2}}-V_{q}^{\infty} \sum_{i=e, h} \Pi_{i}(q, \omega)$,

with $V_{q}^{\infty}=V_{q} / \epsilon_{\infty}$ is the bare Coulomb potential scaled by the high-frequency dielectric constant, and $\Pi_{i}(q, \omega)$ the Lindhard function for a Q1D system of electrons or holes $(i=e$ for electrons and $i=h$ for holes). We also include a phenomenological decay parameter $\gamma=7 \mathrm{ps}^{-1}$ (inverse of LO-phonon lifetime) in the phonon part of the dielectric function. The energy-loss rate (or power loss) of hot-electrons in an interacting system of electron-hole-phonon system is

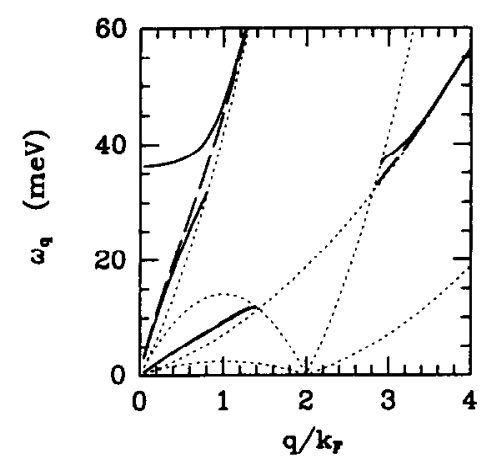

Fig. 1. Coupled and uncoupled mode dispersion relations for a Q1D electron-hole-LO-phonon system at $T=0$. The thin dotted lines indicate the single-particle continua of electrons and holes assumed at the same density $N=10^{6} \mathrm{~cm}^{-1}$. The solid lines show the coupled modes whereas the dashed lines are for uncoupled modes.

given by

$$
\begin{aligned}
\frac{\mathrm{d} E_{k}}{\mathrm{~d} t}= & \int \frac{\mathrm{d} q}{2 \pi} \int \mathrm{d} \omega \omega P(q, \omega) \delta\left(\omega+k q / m-q^{2} / 2 m\right) \\
& \times[1-f(k-q)] .
\end{aligned}
$$

\section{RESULTS AND DISCUSSION}

We now illustrate and discuss our results. The system we consider is a circular GaAs quantum wire of radius $R=50 \AA$, and plasma density $n=5 \times$ $10^{5} \mathrm{~cm}^{-1}$ such that only the lowest electron and hole subbands are occupied. We use the material parameters $\omega_{\mathrm{TO}}=33.8 \mathrm{meV}, \omega_{\mathrm{LO}}=36.5 \mathrm{meV}$, and $\epsilon_{0}=12.9$ for GaAs, and take the electron and hole effective masses to be $m_{e}=0.067 \mathrm{~m}$ and $m_{h}=0.5 \mathrm{~m}$. For the Q1D bare Coulomb interaction $V_{q}$ we employ the model developed by Gold and Ghazali [10]. The undamped coupled modes (solid lines) of the system at $T=0$ are obtained from $\operatorname{Re}[\epsilon(q, \omega ; T)]=0$, and these are illustrated in Fig. 1 together with the uncoupled intrasubband collective modes (dashed lines) for comparison. We note that the uncoupled collective modes of an electron-hole system do not have Landau damping at zero temperature. Besides the ordinary plasma mode, there exists a second acousticplasma mode for frequencies above the single-particle excitations of the heavy particle (holes), and below the single-particle excitations of the light particle (electrons) [11].

The momentum relaxation rate $\Gamma_{k}$ for the electronhole system (solid line) and for the coupled system of LO-phonons and neutral plasma (dotted line) at $T=0$ is shown in Fig. 2(a). We observe that in the absence of LO-phonons, scattering is predominantly from plasmons due to the electron gas. As the dotted line 

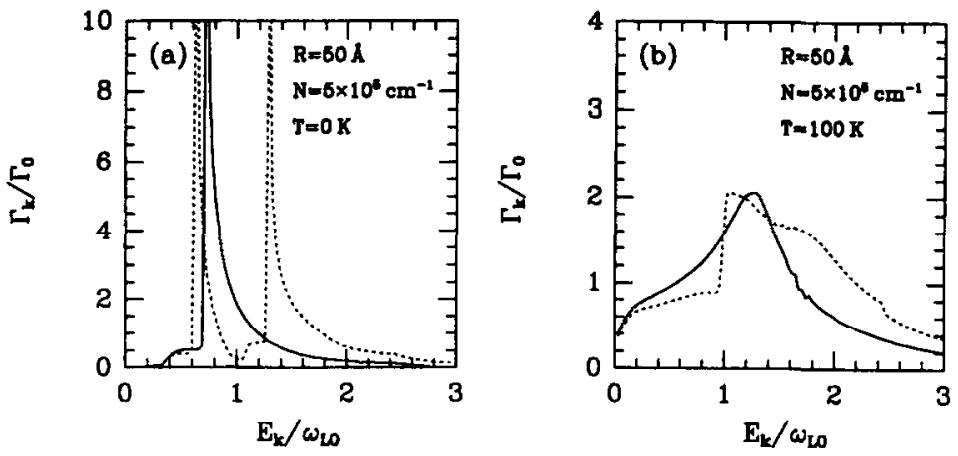

Fig. 2. (a) Momentum relaxation rate $\Gamma_{k}$ as a function of the hot-electron energy at $T=0$. (b) Same as (a) for $T=100 \mathrm{~K} . \Gamma_{0}=8.7 \mathrm{ps}^{-1}$. The solid lines denote scattering from electron-hole plasma (without phonons), whereas the dotted lines denote scattering from the coupled system of electron-hole plasma and LO-phonons.

indicates, the coupled mode system affects the relaxation rate significantly. There are two thresholds yielding a large $\Gamma_{k}$. The low energy threshold is due to the ordinary plasmons (collective excitations in the absence of holes and phonons), and the second one is due to the LO-phonon-like mode (at a slightly higher value than $E_{k}=\omega_{\text {LO }}$ due to coupling). The acoustic plasmons as they exit the particle-hole band give rise to the low energy bump. In general, collective excitations of the coupled mode system give the dominant contribution to $\Gamma_{k}$ in Q1D structures $[6,7]$. The effect of finite temperature is to smooth the observed peaks in $\Gamma_{k}$ [Fig. 2(b)], and at room temperature results look similar to those obtained by assuming bare LO-phonons (not shown). The results obtained here for the momentum relaxation rates indicate behavior different than the $2 \mathrm{D}$ and $3 \mathrm{D}$ structures $[4,12]$. As the growth technology and fabrication techniques for Q1D systems become more refined, our predictions could be experimentally tested [13].

Figure 3 shows the power loss due to emission and absorption of phonons, plasmons and coupled plasmon-phonon modes denoted by the dashed, solid, and dotted lines, respectively, for varying temperatures. At zero temperature the optical phonons can only interact for $E=\omega_{\mathrm{LO}}$ because of the damping (LO-
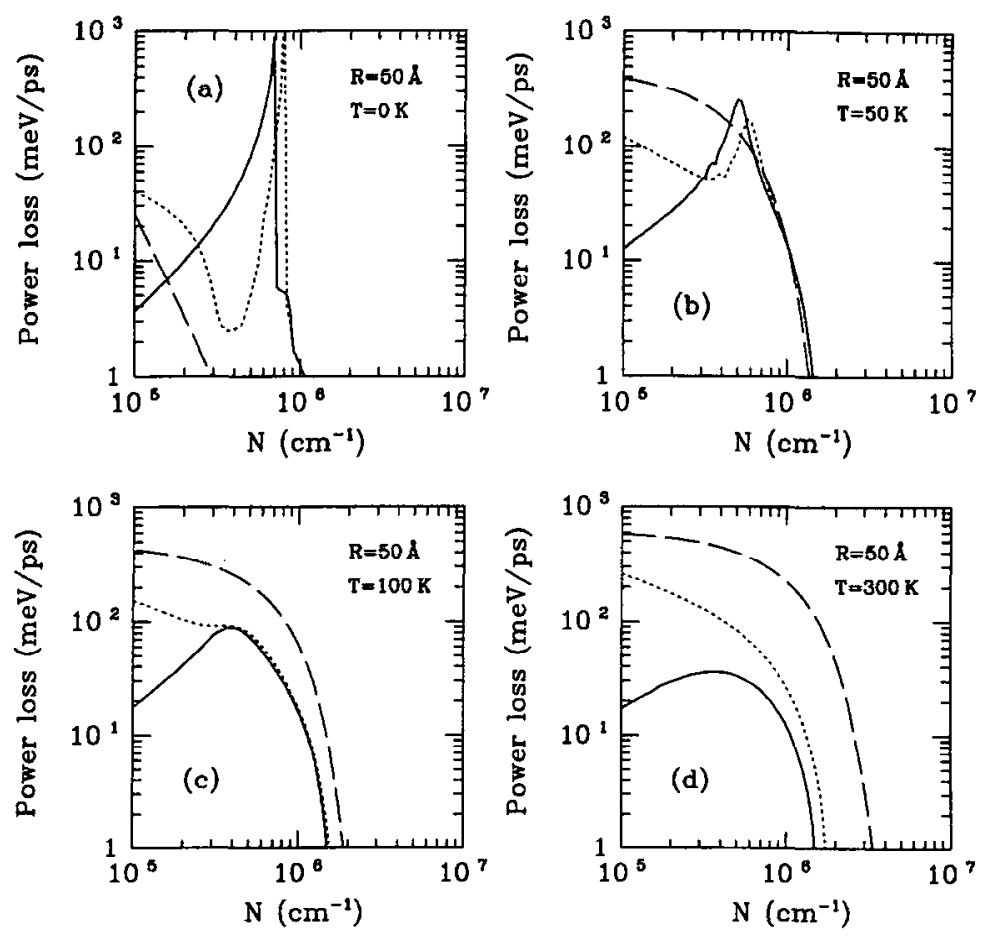

Fig. 3. Power loss for $E_{k}=\omega_{\mathrm{LO}}$ electron, as a function of plasma density. Solid, dashed, and dotted lines indicate plasmons, phonons, and coupled plasmon-phonon modes. (a) $T=0$, (b) $T=50 \mathrm{~K}$, (c) $T=100 \mathrm{~K}$, and (d) $T=300 \mathrm{~K}$. 
phonon lifetime) included in $\epsilon_{T}(q, \omega)$. This means that firstly the result is not infinite and secondly that although the final state would not be empty for the electron if it emitted a phonon of energy $\omega_{\mathrm{LO}}$, it can now emit phonons of slightly different energies. As the density increases the power loss drops because of the decrease in the likelihood of emission and also the lowering of the energy change. The sharp change at around $N=10^{6} \mathrm{~cm}^{-1}$ for the power loss due to plasmons is because of the sudden lack of conservation of energy and momentum when interacting with the electron gas collective modes. These modes are lowered in energy when coupled to the phonons (see Fig. 1) so it is expected that this sudden change will occur at a higher density in the coupled mode case.

As the temperature is increased the plasmon-like modes become damped by single particle excitations, thus no sudden changes occur. Also, these modes increase in energy with increasing temperature so the maximum power loss due to these modes should occur at lower densities. Because the phonon-like mode also becomes damped the overall power loss, where this mode contributes, drops below that of just phonons [Fig. 3(b)]. As the temperature is increased, and the plasmon-like mode becomes indistinguishable, the power loss becomes more like that for just phonons although somewhat reduced due to the screening which occurs at this energy [Fig. 3(c) and (d), see also the graph for momentum relaxation rates where the rate for coupled modes is less than that for just phonons]. The high temperature case is where the static screening limit should be valid.

In summary, we have considered the scattering of a hot-electron from a coupled electron-hole-phonon system in quantum wires. In particular, we have studied the density and temperature dependence of momentum relaxation rate and power loss. Since the static approximation is known to overestimate the screening effects, we used a dynamic screening approach. The influence of coupling of phonons to the collective modes of an electron-hole system on the relaxation and energy loss rates is investigated.

Acknowledgements-We would like to thank B.K. Ridley and M. Babiker for useful discussions. This work is supported by the British Council through its Academic Link Scheme. The financial support of EPSRC via a studentship (C.R.B.) and under grant GR/J80269 (N.C.C.) is gratefully acknowledged.
Partial support by the Scientific and Technical Research Council of Turkey (TUBITAK) under Grant No. TBAG-AY/77 is also acknowledged (B.T.).

\section{REFERENCES}

1. Levi, A.F.J., Hayes, J.R., Platzman, P.M. and Weigmann, W., Phys. Rev. Lett. 55, 2071 (1985); Heiblum, M., Galbi, D. and Weckwerth, M., Phys. Rev. Lett. 62, 1057 (1989); for a review see also Mirlin, D.N. and Perel, V.I., Spectroscopy of Nonequilibrium Electrons and Phonons, (Edited by C.V. Shank and B.P. Zakharchenya). North-Holland, Amsterdam (1992).

2. Rorison, M. and Herbert, D.C., J. Phys. C19, 6357 (1986); Beton, P.H., Long, A.P. and Kelly, M.J., Solid State Electron. 31, 637 (1988); Jalabert, R. and Das Sarma, S., Phys. Rev. B41, 3651 (1990).

3. Hu, B.Y.-K. and Das Sarma, S., Phys. Rev. B44, 8319 (1991); Semicond. Sci. Technol. 7, B305 (1992).

4. Young, J.F. and Kelly, P.J., Phys. Rev. B47, 6316 (1993); Young, J.F., Henry, N.L. and Kelly, P.J., Solid State Electron. 32, 1567 (1989); Kash, J.A., Phys. Rev. B40, 3455 (1989).

5. Hu, B.Y.-K. and Das Sarma, S., Appl. Phys. Lett. 61, 1208 (1992); Phys. Rev. B48, 5469 (1993).

6. Hwang, E.H. and Das Sarma, S., Phys. Rev. B52, 8668 (1995).

7. Bennett, C.R., Constantinou, N.C. and Tanatar, B., J. Phys. Condens. Matter. 7, L669 (1995).

8. Das Sarma, S., Jain, J.K. and Jalabert, R., Phys. Rev. B41, 3561 (1990); Dharma-wardana, M.W.C., Phys. Rev. Lett. 66, 197 (1991); Phys. Rev. Lett. 67, 2917 (1991); Lei, X.L. and Wu, M.W., Phys. Rev. B47, 13338 (1993).

9. Platzman, P.M. and Wolff, P.A., Waves and Interactions in Solid State Plasmas, Solid State Physics, Supplement 13, p. 47. Academic Press, New York, 1973; Pines, D. and Nozieres, P., The Theory of Quantum Liquids. W.A. Benjamin, New York, 1966; Mahan, G.D., Many Particle Physics. Plenum, New York, 1981; Ridley, B.K., Quantum Processes in Semiconductors, 3rd ed., OUP, Oxford, 1993.

10. Gold, A. and Ghazali, A., Phys. Rev. B41, 7626 (1990).

11. Tanatar, B., Solid State Commun. 92, 699 (1994).

12. Jalabert, R. and Das Sarma, S., Solid State Electron. 32, 1989, 1259; Phys. Rev. B40, 9723 (1989).

13. Maciel, A.C., Kiener, C., Rota, L., Ryan, J.F., Marti, U., Martin, D., Morier-Gemoud, F. and Reinhart, F.K., Appl. Phys. Lett. 66, 3039 (1995). 